\title{
The transcriptional network of WRKY53 in cereals links oxidative responses to biotic and abiotic stress inputs
}

\author{
Leon Van Eck • Rebecca M. Davidson • Shuchi Wu • \\ Bingyu Y. Zhao • Anna-Maria Botha • Jan E. Leach • \\ Nora L. V. Lapitan
}

Received: 21 January 2014 /Revised: 14 April 2014 / Accepted: 16 April 2014 /Published online: 29 April 2014

(C) The Author(s) 2014. This article is published with open access at Springerlink.com

\begin{abstract}
The transcription factor WRKY53 is expressed during biotic and abiotic stress responses in cereals, but little is currently known about its regulation, structure and downstream targets. We sequenced the wheat ortholog TaWRKY53 and its promoter region, which revealed extensive similarity in gene architecture and cis-acting regulatory elements to the rice ortholog OsWRKY53, including the presence of stressresponsive abscisic acid-responsive elements (ABRE) motifs and GCC-boxes. Four proteins interacted with the WRKY53 promoter in yeast one-hybrid assays, suggesting that this gene can receive inputs from diverse stress-related pathways such as calcium signalling and senescence, and environmental cues such as drought and ultraviolet radiation. The Ser/Thr receptor kinase ORK10/LRK10 and the apoplastic peroxidase POC1 are two downstream targets for regulation by the WRKY 53
\end{abstract}

Electronic supplementary material The online version of this article (doi:10.1007/s10142-014-0374-3) contains supplementary material, which is available to authorized users.

L. Van Eck • N. L. V. Lapitan $(\bowtie)$

Department of Soil and Crop Sciences, Colorado State University, Fort Collins, CO 80523, USA

e-mail: nora.lapitan@colostate.edu

L. Van Eck $\cdot$ A.-M. Botha

Department of Genetics, Stellenbosch University, Stellenbosch,

Western Cape 7600, South Africa

R. M. Davidson · J. E. Leach

Department of Bioagricultural Sciences and Pest Management,

Colorado State University, Fort Collins, CO 80523, USA

R. M. Davidson

Integrated Center for Genes, Environment \& Health,

National Jewish Health, Denver, CO 80206, USA

S. Wu • B. Y. Zhao

Department of Horticulture, Virginia Tech, Blacksburg,

VA 24061, USA transcription factor, predicted based on the presence of W-box motifs in their promoters and coregulation with WRKY53, and verified by electrophoretic mobility shift assay (EMSA). Both ORK10/LRK10 and POC1 are upregulated during cereal responses to pathogens and aphids and important components of the oxidative burst during the hypersensitive response. Taken with our yeast two-hybrid assay which identified a strong protein-protein interaction between microsomal glutathione $S$-transferase 3 and WRKY53, this implies that the WRKY53 transcriptional network regulates oxidative responses to a wide array of stresses.

Keywords Triticum aestivum - Oryza sativa · Plant disease resistance . WRKY transcription factors - Gene regulation $\cdot$ Protein-DNA interactions

\section{Introduction}

WRKY53 is a WRKY transcription factor integral to several biotic and abiotic stress resistance responses in cereals such as wheat (Triticum aestivum L.) and rice (Oryza sativa L.). The rice ortholog, OsWRKY53, is expressed in roots and leaves and is inducible by drought stress and chitinous elicitors (Akimoto-Tomiyama et al. 2003; Ramamoorthy et al. 2008). The wheat ortholog, TaWRKY53, is induced during leaf senescence (Wu et al. 2008) and infestation by the Russian wheat aphid, Diuraphis noxia Kurdjumov (Botha et al. 2010; Smith et al 2010). Overexpression of OsWRKY53 induces pathogenesis-related (PR) protein expression and greatly reduces symptoms of infection by the rice blast fungus, Magnaporthe oryzae (Hebert) Barr (Chujo et al. 2007; Marcel et al. 2010), whereas silencing of TaWRKY53 results in suppression of the oxidative burst and increased aphid susceptibility (Van Eck et al. 2010). 
The recent systematic census and phylogenetic analysis of 92 WRKY transcription factors in wheat by Zhu et al. (2013) has greatly expanded our knowledge of this transcription factor family and its role in stress regulation in this species. However, apart from some EST information (Wu et al. 2008), almost nothing is known about the structure and function of TaWRKY53. Upstream and downstream components of the WRKY53 transcriptional network have also not been identified in either wheat or rice. Considering the prominent role of WRKY53 in biotic (Chujo et al. 2007; Van Eck et al. 2010) and abiotic stress (Ramamoorthy et al. 2008; Wu et al. 2008) and thus its potential as a target for plant improvement, we characterized the structure of TaWRKY53 and its promoter region and then identified upstream and downstream genetic components of the WRKY53 transcriptional network of the cereals wheat and rice.

\section{Experimental procedures}

TaWRKY53 promoter isolation and characterization

Wheat genomic DNA isolated from the D. noxia-resistant cv. 'Gamtoos-R' (GR) (Van Eck et al. 2010) was employed in genome walking using the GenomeWalker Universal Kit (Clontech, Mountain View, CA, USA) according to the manufacturer's instructions. Nested primers specific to the 5 ' end of the TaWRKY53 coding sequence (CDS) were designed based on accession EF368357, a WRKY53 cDNA clone isolated from hexaploid wheat (T. aestivum L.) cv. 'Nongda 3338' (Wu et al. 2008). Primary and secondary digest library amplifications were performed using LongAmp Taq (New England Biolabs, Ipswich, MA, USA) and nested adaptorspecific primers and gene-specific primers TaWRKY53 GSP1 and TaWRKY53 GSP2 (Table 1). The following cycling parameters were used: initial denaturation at $94{ }^{\circ} \mathrm{C}$ for $30 \mathrm{~s} ; 7$ amplification cycles consisting of denaturation at $94{ }^{\circ} \mathrm{C}$ for $25 \mathrm{~s}$, annealing and extension at $72{ }^{\circ} \mathrm{C}$ for $3 \mathrm{~min} ; 32$ amplification cycles consisting of denaturation at $94{ }^{\circ} \mathrm{C}$ for $25 \mathrm{~s}$, annealing and extension at $67{ }^{\circ} \mathrm{C}$ for $3 \mathrm{~min}$; final extension at $67^{\circ} \mathrm{C}$ for $7 \mathrm{~min}$. The presence of amplification products was verified by agarose gel electrophoresis. Individual amplicons were gel purified, cloned into the pGEM-T Easy vector (Promega, Madison, WI, USA) and sequenced. Sequence reads obtained from genome walking were assembled into contigs using Geneious Pro 5.4 (Drummond et al. 2011). Promoter characterization was performed using a combination of the cis-acting regulatory element databases PLACE (Higo et al. 1999) and PlantCARE (Lescot et al. 2002), and manual scans for the presence of putative W-boxes using the $(\mathrm{C} / \mathrm{T}) \mathrm{TGAC}(\mathrm{C} / \mathrm{T})$ consensus sequence.

\section{TaWRKY53 CDS and gene model}

The CDS of TaWRKY53 was amplified out of cDNA and genomic DNA from GR wheat with primers TaWRKY53_CDS_fwd and TaWRKY53_CDS_rvs (Table 1). The following cycling parameters were used: initial denaturation at $94{ }^{\circ} \mathrm{C}$ for $1 \mathrm{~min} ; 37$ amplification cycles consisting of denaturation at $94{ }^{\circ} \mathrm{C}$ for $20 \mathrm{~s}$, annealing at $60{ }^{\circ} \mathrm{C}$ for $20 \mathrm{~s}$, extension at $65^{\circ} \mathrm{C}$ for $1: 40$; final extension at $65^{\circ} \mathrm{C}$ for $7 \mathrm{~min}$. PCR products were cloned into the pGEM-T Easy vector and sequenced. Sequences were assembled and aligned with GenBank accessions EF368357 and EF368364 to confirm their identity.

\section{TaWRKY53 promoter DNA-protein interaction assays}

Proteins interacting with the TaWRKY53 promoter were identified in yeast one-hybrid assays using Gateway-based DNA bait and protein expression prey vectors (Deplancke et al. 2004). The $1.2-\mathrm{kb}$ promoter region of TaWRKY53 was amplified in three segments, using PCR primers attB4-Pw53400 fwd and attB1R-Pw53-400 rvs, attB4-Pw53-800 fwd and attB1R-Pw53-800 rvs, and attB4-Pw53-1200 fwd and attB1R-Pw53-1200 rvs (Table 4) to generate $-400: \mathrm{P}_{W 53}$, $-800: \mathrm{P}_{W 53}$ and $-1200: \mathrm{P}_{W 53}$ with added Gateway attB transposition sites. The following cycling parameters were used: initial denaturation at $94^{\circ} \mathrm{C}$ for $2 \mathrm{~min} ; 40$ amplification cycles consisting of denaturation at $94{ }^{\circ} \mathrm{C}$ for $30 \mathrm{~s}$, annealing at $63{ }^{\circ} \mathrm{C}$ for $30 \mathrm{~s}$ and extension at $65{ }^{\circ} \mathrm{C}$ for $50 \mathrm{~s}$; final extension at
Table 1 Primers for the characterization of WRKY53 in wheat

\begin{tabular}{lll}
\hline Purpose & Primer ID & Primer sequence \\
\hline Genome walking & TaWRKY53_GSP1 & CGCCAGACCCTGATAGAAGCTCAGTCAAGG \\
& TaWRKY53_GSP2 & AAGGAGGACATGGCGATCGACGCGACGGAA \\
Full-length clones & TaWRKY53_CDS_fwd & CCCTGCTCCTCCCGTCGCTC \\
& TaWRKY53_CDS_rvs & CGTGGACCCACATGTAAACGCCA \\
Protein expression & TaWRKY53exp_fwd & ATGTCCTCCTCCACGGGGAGCTTGGACC \\
& TaWRKY53exp_rvs & GCCGCGGCCTAGCCTGCCTAGCTAGCAG \\
\hline
\end{tabular}


$65{ }^{\circ} \mathrm{C}$ for $10 \mathrm{~min}$. The $a t t B$ PCR products were gel purified, recombined with the pDONR P4-P1R vector (Invitrogen, Carlsbad, CA, USA) in a BP clonase reaction, transformed into chemically competent DH5 $\alpha$ Escherichia coli, and selected for on LB media containing $20 \mu \mathrm{g} \mathrm{mL}^{-1}$ kanamycin. Recombinant entry clones were isolated and recombined with the pDEST-HIS3 destination vector in separate LR clonase reactions to form three expression clones, which were selected for on LB media containing $100 \mu \mathrm{g} \mathrm{mL} \mathrm{m}^{-1}$ ampicillin. All expression clones were sequenced to verify insert identity. YM4271 yeast cells were transformed with the corresponding pDEST-HIS3 expression clones to generate three distinct DNA bait strains placing the HIS3 reporter gene under the control of the $-400: \mathrm{P}_{W 53},-800: \mathrm{P}_{W 53}$ or $-1200: \mathrm{P}_{W 53}$ promoter segments. pDEST-HIS3 expression clones were linearized with $\mathrm{XhoI}$ restriction endonuclease prior to transformation to assist chromosomal integration of the bait constructs at the YM4271 his3-200 locus. Recombinant clones were selected on SD/-His/-Ura double dropout (DDO) media. To test autoactivation of the HIS3 reporter gene and cytotoxicity of the clones, 20 yeast colonies from each double bait strain were replica plated onto $\mathrm{SD} /-$ His/-Ura media supplemented with 0 , $25,50,75$ or $100 \mathrm{mM} \mathrm{3-amino-1,2,4-triazole} \mathrm{(3-AT)} \mathrm{and}$ colony growth was monitored. Colonies that exhibited minimal growth at the lowest possible 3-AT concentration were selected as suitable DNA bait strains for yeast one-hybrid assays. All colonies derived from $-800: \mathrm{P}_{W 53}$ and $-1200: \mathrm{P}_{W 53}$ exhibited high levels of autoactivation in the yeast one-hybrid system; therefore, these promoter elements were not further analyzed. The protein expression prey library consisted of pACTGW-attR prey vectors with in-frame Nterminal fusions of activation domain (AD) to a previously constructed rice biotic stress cDNA library. This library was created from $O$. sativa ssp. japonica cv. 'Nipponbare' inoculated with either of the two bacterial pathogens Xanthomonas oryzae pv. oryzae and X. oryzae pv. oryzicola and incubated for varying lengths of time before mRNA isolation (Niño-Liu et al. 2005). Since aphid feeding induces plant responses that overlap with those induced by fungal pathogen attack (Botha et al. 2005; Kaloshian and Walling 2005; Moran and Thompson 2001; Rodriguez and Bos 2013), and since WRKY53 is involved in both aphid and pathogen resistance responses (Chujo et al. 2007; Van Eck et al. 2010), this biotic stress-induced library could be exploited in our yeast-hybrid analysis to find interactors involved in diverse biotic stress responses. The $-400: \mathrm{P}_{W 53}$ DNA bait strain was transformed with the prey vector library and selected for on SD/-His/-Leu/ -Ura triple dropout (TDO) media supplemented with $60 \mathrm{mM}$ 3 -AT. AD vector plasmids were rescued from yeast clones showing positive interactions, subcloned into $\mathrm{DH} 5 \alpha$ E. coli in order to obtain a higher yield and tested for the presence of cDNA inserts by PCR with AD_fwd and AD_rvs primers (Table 4) before being sequenced.
TaWRKY53 protein-protein interaction assays

Proteins interacting with TaWRKY53 were identified in yeast two-hybrid assays using Gateway-based bait and prey vectors expressing the Saccharomyces cerevisiae GAL4 binding domain (BD) and activation domain (AD), respectively (Nakayama et al. 2002). The same biotic stress-induced pACTGW-attR prey vector library was utilized as described for yeast one-hybrid assays. The pASGW-attR bait vector consisted of an in-frame N-terminal fusion of BD to a truncated version of the TaWRKY53 coding sequence lacking the first 180 amino acids to prevent autoactivation (Lai et al. 2011). A clone of TaWRKY53 was amplified from GR cDNA with primers attB1-tW53_fwd and attB2-W53_rvs (Table 4) and $a t t B$ sites attached to either end via PCR. The following cycling parameters were used: initial denaturation at $94{ }^{\circ} \mathrm{C}$ for $30 \mathrm{~s} ; 35$ amplification cycles consisting of denaturation at $94{ }^{\circ} \mathrm{C}$ for $20 \mathrm{~s}$, annealing at $60{ }^{\circ} \mathrm{C}$ for $20 \mathrm{~s}$, extension at $64{ }^{\circ} \mathrm{C}$ for $1: 40$; final extension at $64{ }^{\circ} \mathrm{C}$ for $10 \mathrm{~min}$. The resulting 884 bp attB PCR fragment was cloned into the pDONR 221 donor vector (Invitrogen) via a BP clonase transposition reaction, forming an entry clone, which was transformed into competent $\mathrm{DH} 5 \alpha$ cells and selected for on LB media containing $20 \mu \mathrm{g} \mathrm{mL}{ }^{-1}$ kanamycin. This entry clone was subsequently isolated and recombined with the pASGW-attR destination vector in an LR clonase reaction to form the final pASGW::tW53 expression clone, which was transformed into competent $\mathrm{DH} 5 \alpha$ cells and selected for on LB media containing $100 \mu \mathrm{g} \mathrm{mL}^{-1}$ ampicillin. The expression clone was sequenced to verify the integrity of the reading frame and tested for autoactivation and cytotoxicity by transforming into Y2HGold yeast cells using the Frozen-EZ Yeast Transformation II kit (Zymo Research, Orange, CA, USA), and plating onto SD/-Trp single dropout media supplemented with either $20 \mathrm{ng} \mathrm{mL}^{-1} \mathrm{X}$ - $\alpha$-gal (Gold Biotechnology, St. Louis, MO, USA) or X- $\alpha$-gal and $125 \mathrm{ng} \mathrm{mL}{ }^{-1}$ Aureobasidin A (Clontech). After co-transformation of $1 \mu \mathrm{g}$ each of bait and prey vector into Y2HGold yeast cells, the cells were grown on SD/-Leu/-Trp double dropout media supplemented with $\mathrm{X}$ - $\alpha$-gal (DDOX). Blue colonies were selected and replica plated onto SD/-Ade/-His/-Leu/-Trp quadruple dropout media supplemented with $\mathrm{X}-\alpha$-gal and Aureobasidin A (AurA) (QDOXA). This selects for the presence of BD vector (-Trp), AD vector (-Leu) and the activation of the four reporter genes HIS3 (-His), ADE2 (-Ade), MEL1 (X- $\alpha$-gal) and $A U R 1-C$ (AurA). AD vector plasmids were rescued from yeast clones showing positive interactions by scraping colonies from plates into $67 \mathrm{mM}$ of $\mathrm{KH}_{2} \mathrm{PO}_{4}$ and digesting with $30 \mathrm{U}$ of zymolase (Seikagaku, Tokyo, Japan) for $1 \mathrm{~h}$ at $37^{\circ} \mathrm{C}$. Digestion was followed by column purification with a QIAprep Spin Miniprep kit (Qiagen, Hilden, Germany). Isolated plasmids were subcloned into DH5 $\alpha$ E. coli in order to obtain a higher yield and tested for the 
presence of cDNA inserts by PCR with $\mathrm{AD}$ fwd and AD_rvs primers (Table 4 ) before being sequenced.

\section{Identification of potential WRKY53 targets}

Potential target promoters for the TaWRKY53 transcription factor were identified by mining the rice genome for genes with putative functional linkages to LOC_Os05g27730 (OsWRKY53) via the RiceNet probabilistic functional gene network (Lee et al. 2011). The Gene Coexpression Analysis tool from the MSU Rice Genome Annotation Project Database (Childs et al. 2011) was used to identify genes with expression profiles correlated to that of OsWRKY53 during an infection time course with the hemibiotrophic fungal pathogen M. oryzae (Marcel et al. 2010). Gene Ontology (GO) SLIM assignments for all predicted rice genes were obtained from the MSU Rice Genome Annotation Project (http://rice. plantbiology.msu.edu/downloads.shtml). GO term enrichment tests were performed using a modified Fisher's exact test, and modified EASE scores were calculated to indicate significant enrichment of GO terms in the coregulated genes $(n=96)$ compared to the genome background $(n=67,393)$ (Hosack et al. 2003; Huang et al. 2008). The $1 \mathrm{~kb}$ upstream promoter regions of all predicted gene models in the MSU Rice Genome Annotation Project v6. 1 were screened for the presence of the W-box $(\mathrm{C} / \mathrm{T})$ TGAC $(\mathrm{C} / \mathrm{T})$ consensus motif using a custom Perl script, and numbers of W-box motifs per gene model were calculated. Frequency distributions of numbers of W-box motifs per gene model were calculated and compared between the coregulated gene subset and the genome-wide background with a MannWhitney-Wilcoxon test $(p$ value $=3.288 \mathrm{e}-13)$.

\section{TaWRKY53 protein expression}

The wheat WRKY53 protein was expressed with the Champion $\mathrm{pET}$ SUMO protein expression system (Invitrogen) for use in electrophoretic mobility shift assays (Panavas et al. 2009). The TaWRKY53 CDS was amplified with primers TaWRKY53exp_fwd1 and TaWRKY53_exp_rvs1 (Table 1) from a cloned full-length cDNA template previously isolated from GR wheat. The purified amplification product was ligated to the pET Sumo vector and transformed into Mach1-T1R chemically competent $E$. coli. Once the recombinant plasmid pET::W53 was isolated and sequenced to verify the Nterminal in-frame fusion of the TaWRKY53 CDS with the SUMO tag, the plasmid was transformed into competent BL21(DE3) E. coli for expression. Fresh LB medium containing $50 \mu \mathrm{g} \mathrm{mL}^{-1}$ kanamycin and $1 \%$ glucose was inoculated at a ratio of 1:50 with overnight culture and grown at $37^{\circ} \mathrm{C}$ until mid-log phase $(\mathrm{OD} 600=0.5)$. Protein expression was induced with $1 \mathrm{mM}$ IPTG and the culture incubated for a further $4.5 \mathrm{~h}$ before bacterial cell lysates were prepared.
SUMO::TaWRKY53 fusion protein was purified using the N-terminal polyhistidine $(6 \times \mathrm{His})$ tag and ProBond $\mathrm{Ni}^{2+}$ chelating resin (Invitrogen), following the manufacturer's hybrid purification protocol to ensure maximum solubility and biological activity. TaWRKY53 encompasses 439 amino acids and is calculated to be a $47.39-\mathrm{kDa}$ protein. Therefore, the SUMO::TaWRKY53 fusion protein is expected to be $\sim 60 \mathrm{kDa}$ in size. Protein yield was determined via a Pierce $660 \mathrm{~nm}$ protein assay (Thermo Scientific, Rockford, IL, USA) and was visualized using $10 \%$ polyacrylamide gel electrophoresis. Protein concentration was adjusted to $500 \mu \mathrm{g} \mathrm{mL}^{-1}$ in $30 \%$ glycerol.

\section{Electrophoretic mobility shift assay}

Three rice genes from different functional categories, each with four or more W-boxes, were selected for in vitro binding assays with the expressed TaWRKY53 protein. Based on the $1 \mathrm{~kb}$ upstream sequence information of these genes, biotinylated double-stranded DNA probes 80 bp in length were synthesized (Integrated DNA Technologies, Coralville, IA, USA) (Table 3). Binding assays were performed using the LightShift Chemiluminescent electrophoretic mobility shift assays (EMSA) kit (Thermo Scientific, Barrington, IL, USA). A total of $500 \mu \mathrm{g}$ of purified TaWRKY53 protein was incubated with each probe in binding buffer ( $10 \mathrm{mM}$ Tris, $50 \mathrm{mM} \mathrm{KCl}, 1 \mathrm{mM}$ dithiotreitol, $\mathrm{pH}$ 7.5) and incubated for $20 \mathrm{~min}$ at room temperature. Protein was either incubated with $0.08 \mathrm{ng}$ of labelled probe alone, or with labelled probe and $200 \mathrm{ng}$ of unlabelled probe. Binding reactions were separated on $6 \%$ acrylamide/ $0.5 \times$ TBE non-denaturing gels, transferred to nylon membranes and blocked, washed and detected according to the manufacturer's instructions. Membranes were placed in a film cassette and exposed to X-ray film for $5 \mathrm{~min}$. An alternative EMSA was also performed (Fig. S3) by using primers (Table S3) to amplify larger, $1-\mathrm{kb}$ rice promoter fragments from the genomic DNA of $O$. sativa ssp. japonica cv. 'Nipponbare'. Approximately $1 \mu \mathrm{g}$ of each promoter fragment was combined with $500 \mu \mathrm{g}$ of protein and incubated in binding buffer $(10 \mathrm{mM}$ Tris, $100 \mathrm{mM} \mathrm{KCl}, 1 \mathrm{mM}$ EDTA, $0.1 \mathrm{mM}$ DTT, $5 \% v / v$ glycerol, $0.01 \mathrm{mg} \mathrm{mL}^{-1} \mathrm{BSA}, \mathrm{pH} 7.5$ ) at room temperature for $20 \mathrm{~min}$ (Hellman and Fried 2007). The reactions were separated on $1 \%$ agarose gel in TAE buffer (Berman et al. 1987) and stained using SYBR Green I nucleic acid gel stain (Invitrogen) according to the manufacturer's instructions.

\section{Results}

WRKY53 sequence features are remarkably well-conserved

We amplified the entire 1,879 nucleotide open reading frame and 1,211 nucleotides of upstream promoter sequence for the 
wheat ortholog of WRKY53 (GenBank accession KC174859). Our primers (Table 1) did not detect multiple transcripts, and LOC_Os05g27730, encoding rice ortholog OsWRKY53, is predicted to produce only a single splicing variant. Aligning the wheat genomic and cDNA sequences allowed for the mapping of intron-exon boundaries and the construction of a gene model for TaWRKY53, which was then compared to the gene model for OsWRKY53 (Fig. 1a). The coding region of TaWRKY53 spans five exons, similar to the intron-exon pattern predicted for OsWRKY53. Exons 3 and 4 encode the two conserved WRKY domains, consistent with its classification as a group Ia WRKY protein (Zhu et al. 2013); exon 3 also includes a zinc finger motif, which conforms to the $\mathrm{CX}_{[4-5]} \mathrm{CX}_{[22-23]} \mathrm{HXH}$ consensus sequence for $\mathrm{Cys}_{2}-\mathrm{His}_{2}-$ type zinc fingers; the last three codons of exon 3 and the first codon of exon 4 encode the nuclear localization signal. Similar to AtWRKY33, the ortholog from Arabidopsis thaliana (Mao et al. 2011), there are several putative phosphorylation sites in the N-terminal region (Fig. S1).

The 1.2-kb upstream promoter sequences of TaWRKY53 and $O s W R K Y 53$ were inspected for the presence of cis-acting regulatory motifs. Although the motifs found are similar between wheat and rice, their number, orientation and distance from the start of translation varies (Fig. 1b). Two abscisic acidresponsive elements (ABRE) present in the TaWRKY53 promoter conform to the $(\mathrm{A} / \mathrm{C}) \mathrm{ACG}(\mathrm{C} / \mathrm{T}) \mathrm{GC}$ motif consensus, at -655 and $-875 \mathrm{bp}$, and a GCC-box ethylene-responsive element conforms to the AGCCGCC motif, at -567 bp upstream. By contrast, the rice promoter has only one ABRE motif, at $-189 \mathrm{bp}$, but two GCC-boxes at -135 and -332 upstream. The TaWRKY53 promoter contains three W-box elements that conform to the $(\mathrm{C} / \mathrm{T}) \mathrm{TGAC}(\mathrm{C} / \mathrm{T})$ consensus motif, at -869 , $-1,064$ and $-1,178$ bp upstream of the ATG translation initiation codon (Fig. 1b). The OsWRKY53 promoter has a similar number of W-boxes, at $-298,-316$ and -322 bp (Chujo et al. 2009).

Proteins interacting with the WRKY53 promoter and the WRKY53 protein

To discover possible transcriptional regulators for WRKY53, and to test whether such interactions are conserved across cereals, constructs were made for yeast one-hybrid assays with three discrete segments of the $1.2 \mathrm{-kb}$ promoter region of TaWRKY53, -400: $\mathrm{P}_{W 53},-800: \mathrm{P}_{W 53}$ and $-1200: \mathrm{P}_{W 53}$ (Fig. 1b). These were used as DNA bait against a previously constructed rice pathogen-responsive cDNA expression library (Niño-Liu et al. 2005). A total of four positive interactors with $-400: \mathrm{P}_{W 53}$ were identified (Fig. 2a): LOC_Os01g72100, encoding OsCML10; LOC_Os08g42850, encoding OsFKBP16-3; LOC_Os07g47640, encoding an ultraviolet B-repressible protein; and LOC_Os04g45834, encoding a DUF584 domaincontaining protein. Because of autoactivation of the $-800: \mathrm{P}_{W 53}$ and $-1200: \mathrm{P}_{W 53}$ DNA bait strains, likely by endogenous yeast proteins, these promoter segments were excluded from our analysis. Sequencing results for the four WRKY 53 promoter interactors are summarized in Table 2. We also investigated protein-protein interactions with WRKY 53 to identify additional components of its transcriptional network. We expressed a truncated TaWRKY 53 protein lacking the first 180 amino acids to prevent autoactivation (Lai et al. 2011). This truncated wheat protein was fused to the GAL4 binding domain for yeast two-hybrid assays against the same rice prey vector library used for yeast one-hybrid analysis. Of more than 200 individual clones obtained, only one clone, 318, maintained strong growth and reporter gene activation upon repeated replica plating on high stringency media

(a)

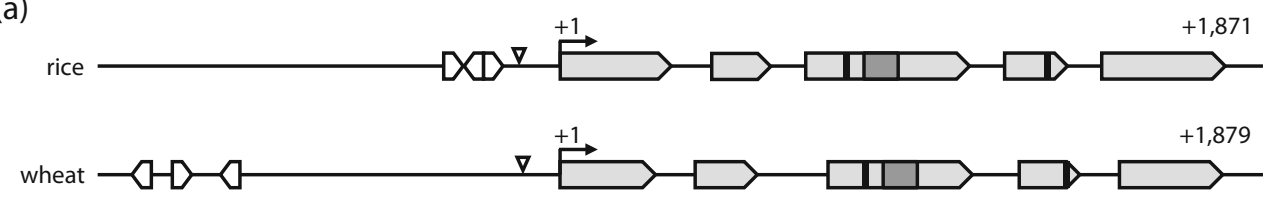

(b)

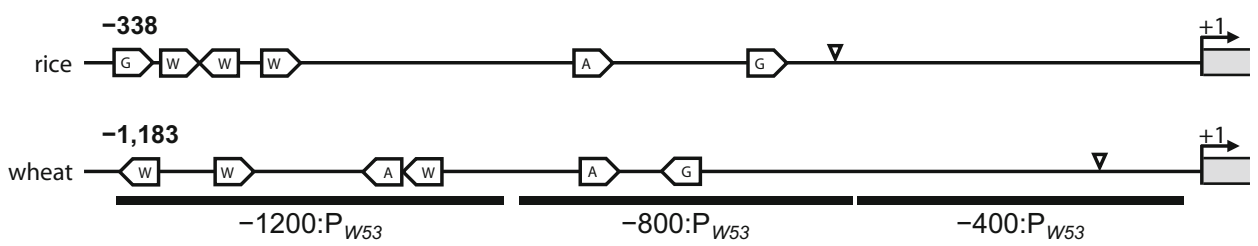

Fig. 1 a Gene models for the WRKY53 orthologs in rice and wheat: both orthologs span five exons, represented by grey arrows; the two conserved WRKY domains are indicated by black bars; the zinc finger motif is indicated by a dark grey bar. The relative promoter positions of the Wbox WRKY transcription factor binding motifs are indicated by open arrows. b Features of the promoter regions of the rice and wheat orthologs of WRKY53. Putative cis-acting regulatory elements are indicated by open arrows: $A$ ABRE abscisic acid-responsive element; $G$ GCC-box ethylene-responsive element; $W$ W-box WRKY transcription factor binding motif. The regions amplified from TaWRKY53 for use in yeast one-hybrid assays are indicated by horizontal black bars 
(Fig. 2b). We speculate that this high level of stringency (requiring the activation of four separate reporter genes) could explain why only a single clone was obtained. However, a bait protein from wheat may bind to rice proteins less efficiently than a rice homolog, and the truncation of that wheat bait protein may also have negatively affected the ability of our assay to discover proteins interacting with WRKY53. The cDNA expressed by 318 had significant homology to LOC_Os03g50130, which encodes a putative microsomal glutathione $S$-transferase 3 (Table 2).

Potential target genes from WRKY53 coexpression networks

Following the rationale that genes that function together have similar expression profiles, the RiceNet Probabilistic Functional Gene Network (Lee et al. 2011) and MSU Gene Coexpression Analysis (Childs et al. 2011) tools were used to identify candidate genes potentially regulated by WRKY53. RiceNet returned 36 loci linked to LOC_Os05g27730 which encodes OsWRKY53 (Fig. S2a), with coherence scores ranging from 1.11 (LOC_Os03g01740) to 3.74 (LOC_Os04g34140). The MSU Gene Coexpression Analysis tool indicated that a total of 62 loci out of 1,161 in the M. oryzae-induced dataset were correlated with OsWRKY53 expression at a very stringent cut-off of between 0.99 and 1 (Fig. S2b). Interestingly, only four loci (LOC_Os09g37080,
LOC_Os03g53020, LOC_Os03g01740 and LOC_Os01g38980) were present in both analyses. Two defence-related genes upregulated in OSWRKY53-overexpressing transgenic rice cells (Chujo et al. 2007) were also included to generate a combined set of 96 potential targets for WRKY53 (Table S1).

The 1-kb promoters of these WRKY53-coregulated loci are enriched for the presence of W-box WRKY binding motifs when compared to the frequency distribution of all gene models across the rice genome (Fig. 3). This provides further evidence that some of these coregulated genes may be downstream targets of WRKY53. The list of coregulated loci exhibits a significant enrichment for Gene Ontology (GO) terms corresponding to molecular functions such as kinase activity, DNA binding and transcription regulator activity, and biological processes such as response to stress, response to extracellular stimulus, and signal transduction (Table S2), consistent with the function of WRKY53 as a transcription factor involved in stress responses.

To address whether the promoters of bioinformatically determined target genes can be bound by the WRKY 53 protein, and also whether binding specificity is conserved across cereals, we designed DNA probes based on promoter sequences of three coregulated rice genes (Tables 3 and 4). These were used as targets for binding in electrophoretic mobility shift assays (EMSAs) with an expressed wheat

(a)

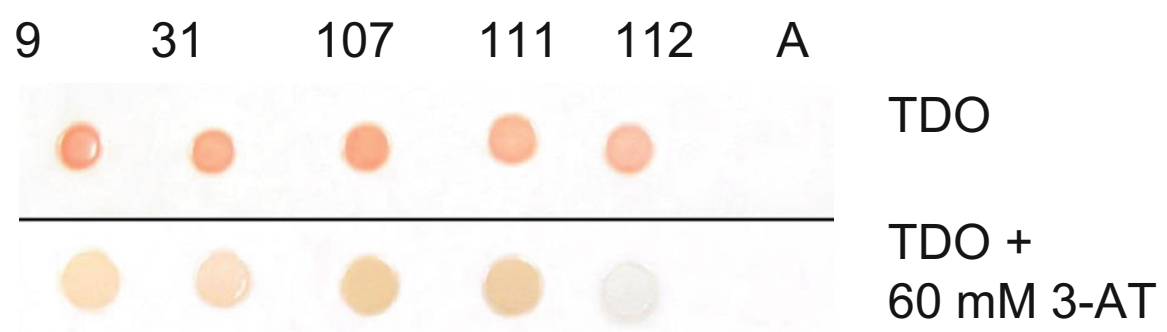

(b)

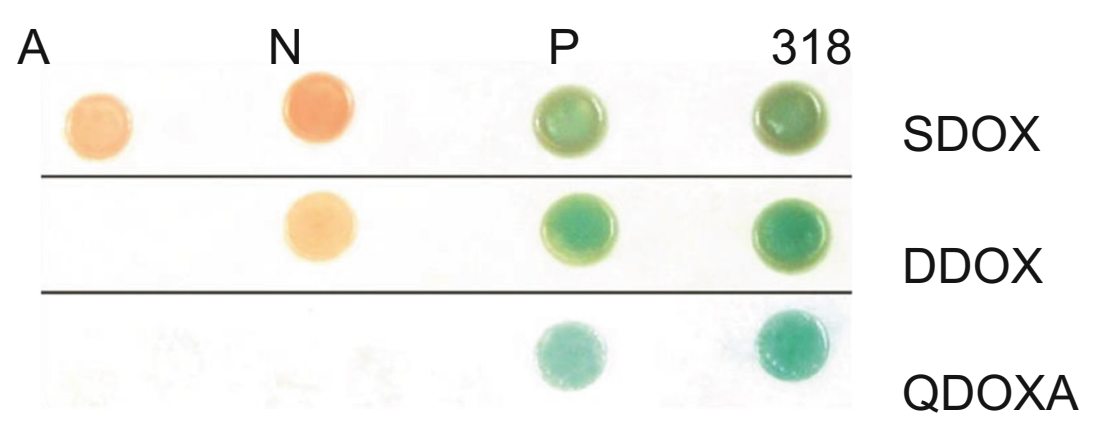

Fig. 2 a Yeast one-hybrid interactions. Colonies 9-111 have the HIS3 reporter gene under the control of the $-400: \mathrm{P}_{W 53}$ promoter segment. Colony 112 harbours an empty prey protein vector and acts as a negative control. The identities of the interactors are summarized in Table 2. A autoactivation control, TDO triple dropout SD/-His/-Leu/-Ura media, 3AT 3-amino-1,2,4-triazole. b Yeast two-hybrid interactions. All colonies express the truncated WRKY53 protein from the pASGW::tW53 vector.
Blue colour indicates the activation of the MEL1 gene. $A$ autoactivation control, $N$ negative control, $P$ positive control; 318 , positive interactor, a microsomal glutathione $S$-transferase. SDOX single dropout $\mathrm{SD} /-\operatorname{Trp} /$ $\mathrm{X}$ - $\alpha$-gal media; $D D O X$ double dropout $\mathrm{SD} /-\mathrm{Leu} /-\operatorname{Trp} / \mathrm{X}-\alpha$-gal media; $Q D O X A$ quadruple dropout $\mathrm{SD} /-\mathrm{Leu} /-\mathrm{Trp} /-\mathrm{Ade} /-\mathrm{His} / \mathrm{X}-\alpha$-gal/AurA media 
Table 2 Yeast-hybrid interactors

\begin{tabular}{lllll}
\hline Interaction & Bait & Clone ID & Homology $^{\mathrm{a}}$ & E-value \\
\hline One-hybrid & $-400: \mathrm{P}_{W 53}$ & 9 & LOC_Os01g72100 OsCML10 calmodulin-related calcium sensor protein & $3.9 \mathrm{e}^{-59}$ \\
& & 31 & LOC_Os08g42850 FKBP-type peptidyl-prolyl cis-trans isomerase & $3.9 \mathrm{e}^{-104}$ \\
& & 107 & LOC_Os07g47640 ultraviolet B-repressible protein & $1.3 \mathrm{e}^{-132}$ \\
& & 111 & LOC_Os04g45834 DUF584 domain-containing protein & $3.1 \mathrm{e}^{-10}$ \\
Two-hybrid & tW53 & 318 & LOC_Os03g50130 microsomal glutathione $S$-transferase 3 & $3.9 \mathrm{e}^{-59}$ \\
\hline
\end{tabular}

${ }^{a}$ Homology based on BLASTn searches of the MSU Rice Genome Annotation Project database

WRKY53 protein (Fig. 4). All three genes selected have distinct functions in plant disease resistance: chitinase-2 (LOC_Os11g47600), a PR (pathogenesis-related) protein expressed in response to biotic stressors such as aphids and pathogenic fungi (Van der Westhuizen et al. 1998; AkimotoTomiyama et al. 2003; Ramonell et al. 2005; Chujo et al. 2007); the Ser/Thr-type receptor kinase ORK 10 (LOC_Os01g02300), induced in cereals infected by biotrophic rust fungi (Feuillet et al. 1997; Cheng et al. 2002; Marcel et al. 2010); and the apoplastic cationic peroxidase POC1 (LOC_Os07g48050), induced by X. oryzae pv. oryzae and aphid infestation as part of the oxidative burst (Young et al. 1995; Van der Westhuizen et al. 1998; Hilaire et al. 2001; Anguelova-Merhar et al. 2002). Binding of the wheat WRKY53 to the promoter fragments of ORK10 and POC1 was observed, but not to the promoter fragment of chitinase-2. An alternative EMSA using longer, 1-kb promoter fragments also did not demonstrate binding of WRKY53 to chitinase-2 (Fig. S3), similar to the lack of binding observed with POX5.1 (LOC_Os07g48040) (Chittoor et al. 1997), a peroxidase related to $P O C 1$ but with no W-boxes in its $1-\mathrm{kb}$ promoter. This might indicate the requirement for additional cofactors or phosphorylation of WRKY53 for binding to the W-boxes in

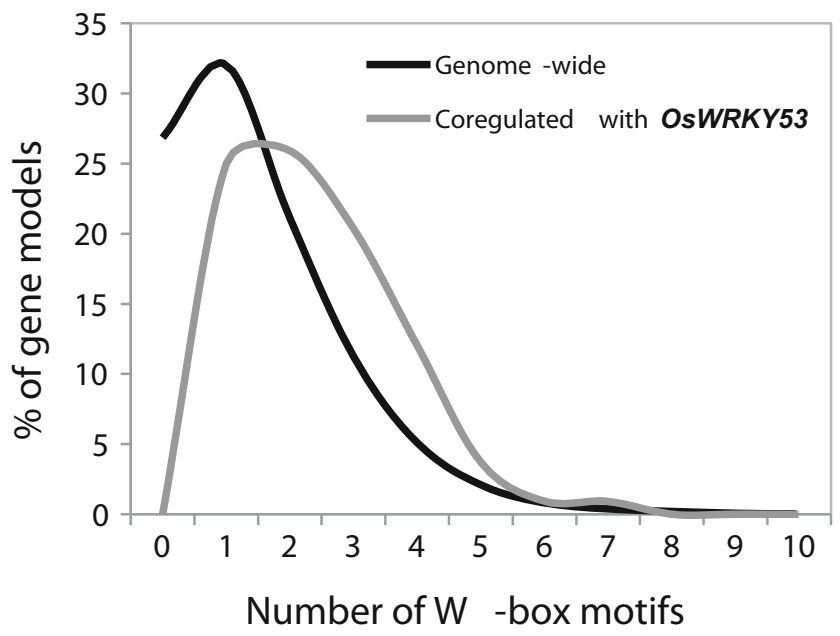

Fig. 3 Frequency distributions of numbers of W-box motifs for all predicted gene models in the MSU rice genome annotation v6.1 $(n=67,393)$ compared to a subset of genes coregulated with OsWRKY53 ( $(n=96)$ the chitinase-2 promoter to occur (Wan et al. 2004; Mao et al. 2011). However, the interaction between wheat WRKY53 and the rice $O R K 10$ and $P O C 1$ promoters in our assay demonstrates that putative genetic interactions determined through bioinformatic analysis can be substantiated by simple in vitro DNA-protein binding assays and that such binding predictions can be translated across related cereals.

\section{Discussion}

The presence of abscisic acid-responsive elements (ABRE) and GCC-box ethylene-responsive elements in the promoter regions of TaWRKY53 and OsWRKY53 (Fig. 1b) supports the function of the WRKY53 transcription factor in stress regulation, since abscisic acid-responsive genes are upregulated during drought (Christmann et al. 2006) and aphid infestation (Park et al. 2006), and GCC-box elements are a hallmark of the promoters of aphid- and pathogen-responsive genes (Rushton and Somssich 1998; Smith and Boyko 2007; Dong et al. 2010). We speculate that similar sets of regulatory factors will be recruited to the promoters of TaWRKY53 and OsWRKY53, although the precise number and position of cis-acting elements might impact binding efficiency and contribute to any presumed interspecific differences between these orthologs. The presence of W-boxes in the TaWRKY53 and OsWRKY53 promoters implies either regulation by other WRKY transcription factors (Rushton et al. 2010; Eulgem and Somssich 2007), or autoregulation as has been described for the orthologs AtWRKY33 in Arabidopsis (Mao et al. 2011) and PcWRKY1 in parsley (Petroselinum crispum L.) (Turck et al. 2004). These $\mathrm{W}$-boxes are required for elicitor responsiveness of OsWRKY53 (Chujo et al. 2009) and pathogen-responsive induction of AtWRKY33 (Lippok et al. 2007). Although the W-boxes are located much further upstream in TaWRKY53, their overall number and orientation resembles that found in orthologs from other plant species (Turck et al. 2004; Lippok et al. 2007). This extensive interspecies preservation of gene architecture and specific regulatory elements is in agreement with phylogenetic evidence (Wu et al. 2008; Zhu et al. 2013) and suggests strong conservation of function and a pivotal role in plant stress responses. It is therefore likely that the rice and 
Table 3 Electrophoretic mobility shift assay probes

\begin{tabular}{lll}
\hline Locus ID & Annotation & EMSA fragment $^{\mathrm{a}}$ \\
\hline LOC_Os11g47600 & chitinase 2 & $\begin{array}{c}\text { AGCCTCACGTTTCGTCCTGATTGCAAGTTGTTGACTTAAATTTGACTTGTCTCGGAAC } \\
\text { AAAACAATAACCTGCAGTCCGT }\end{array}$ \\
LOC_Os01g02300 & ORK10 kinase & $\begin{array}{c}\text { ATCTGGTCAACAATGTATTACACACTGCTTTGACTACTTCCCCCAAAAAAGTACACACT } \\
\text { GCTTTGACTCAGGTCAAACTT }\end{array}$ \\
LOC_Os07g 48050 & POC1 peroxidase & $\begin{array}{c}\text { ACGTAAATTTTTTGAATAAGACAAATGGTCAAACATGTAAGAAAAGAAAGTCAACGGC } \\
\text { GTCATCTATTTAAAAAACGGAT }\end{array}$
\end{tabular}

${ }^{\mathrm{a}}$ The presence of W-boxes is indicated in bold

wheat orthologs of WRKY53 target equivalent sets of stress response genes.

All four proteins that interacted with the WRKY53 promoter in the yeast one-hybrid assays (Table 2) are known to be involved in plant stress responses: OsFKBP16-3 (LOC_Os08g42850), an FKBP-type peptidyl-prolyl cis-trans isomerase (PPI) that functions in osmotic stress tolerance (Ahn et al. 2010; Gollan and Bhave 2010); OsCML10 (LOC_Os01g72100), a member of a calmodulin-related calcium sensor protein family known to interact with transcription factors (Popescu et al. 2007; Reddy and Reddy 2004); an ultraviolet B-repressible protein (LOC_Os07g47640) that serves as a trans-acting negative regulator of stressresponsive genes (Olbrich et al. 2005); and a protein containing a DUF584 domain (LOC_Os04g45834) found in several DNA-binding senescence-related proteins (Fischer-Kilbienski et al. 2010; Krupinska et al. 2002). We therefore conclude that WRKY53 can receive inputs from several stress-related pathways (Fig. 5), including those affording responsiveness to calcium signalling mechanisms initiated by pest and pathogen detection, or to environmental cues such as drought stress and ultraviolet radiation. This is supported by our analyses demonstrating interactions between the WRKY53 promoter and OsCML10, OsFKBP16-3, and an ultraviolet-repressible protein. Evidence from the literature that WRKY53 is highly induced in senescing wheat leaves (Wu et al. 2008) is in accord with our discovery of a protein containing the senescence-related DUF584 domain as a regulator of WRKY53 expression. Additionally, all four of these interactors belong to gene classes that are coregulated with $W R K Y$ genes during various abiotic and biotic stress responses (Izaguirre et al. 2003; Wang et al. 2007; Galon et al. 2008; Qiu et al. 2008; Fischer-Kilbienski et al. 2010).

There is also mounting evidence that glutathione $S$-transferases (GSTs) form an integral part of WRKY transcriptional networks (Hahn and Strittmatter 1994; Olbrich et al. 2005; Shimono et al. 2007; Encinas-Villarejo et al. 2009). This supports our yeast two-hybrid data which indicates that a microsomal glutathione $S$-transferase 3 is able to interact with the WRKY53 protein (Table 2). GSTs play a role in ameliorating oxidative damage (Jakobsson et al. 1999; Gill and Tuteja 2010) and are responsible for scavenging free radicals in the wake of the SA-mediated oxidative burst in response to abiotic stress (Cummins et al. 2011) and pathogen and aphid attack (Lieberherr et al. 2003; Couldridge et al. 2007; Botha et al. 2005; Moloi and van der Westhuizen 2008). Considering that $D$. noxia feeding induces chlorosis and oxidative damage to cereal leaves (Ni et al. 2001; Ni and Quisenberry 2003) and that TaWRKY53 is essential for aphid resistance in wheat (Van Eck et al. 2010), our yeast two-hybrid data provide evidence that membrane-bound glutathione $S$-transferases might alter the reactive oxygen species (ROS) response in a TaWRKY53mediated way. This could be achieved either through the induction of detoxifying gene products to protect the

Table 4 Primers used for the construction of yeast-hybrid vectors

\begin{tabular}{ll}
\hline Primer ID & Primer sequence \\
\hline attB1-tW53_fwd & GGGGACAAGTTTGTACAAAAAAGCAGGCTTATACAATTGGAGGAAGTACGGGCAG \\
attB2-W53_rvs & GGGGACCACTTTGTACAAGAAAGCTGGGTCCTACTAGCAGAGGAGCGACTCGACGAA \\
attB4-Pw53-400_fwd & GGGGACAACTTTGTATAGAAAAGTTGTCTCGATTGATTGCCCGCACCAAA \\
attB1R-Pw53-400_rvs & GGGGACTGCTTTTTTGTACAAACTTGACCGACGGTACATGCCATAGGTCC \\
attB4-Pw53-800_fwd & GGGGACAACTTTGTATAGAAAAGTTGCGTGTTGGTGCAGCCATCTCGTAT \\
attB1R-ppw53-800_rvs & GGGGACTGCTTTTTTGTACAAACTTGTGCGGGGTTTGTTTACTCTGGAA \\
attB4-Pw53-1200_fwd & GGGGACAACTTTGTATAGAAAAGTTGATCAGGGTCTGGCGTAGTCAGGTG \\
attB1R-Pw53-1200_rvs & GGGGACTGCTTTTTTGTACAAACTTGGCATGGTACATCCCCGACCTGAGA \\
AD_fwd & CTATTCGATGATGAAGATACC \\
AD_rvs & GTGAACTTGCGGGGTTTTTCA \\
\hline
\end{tabular}




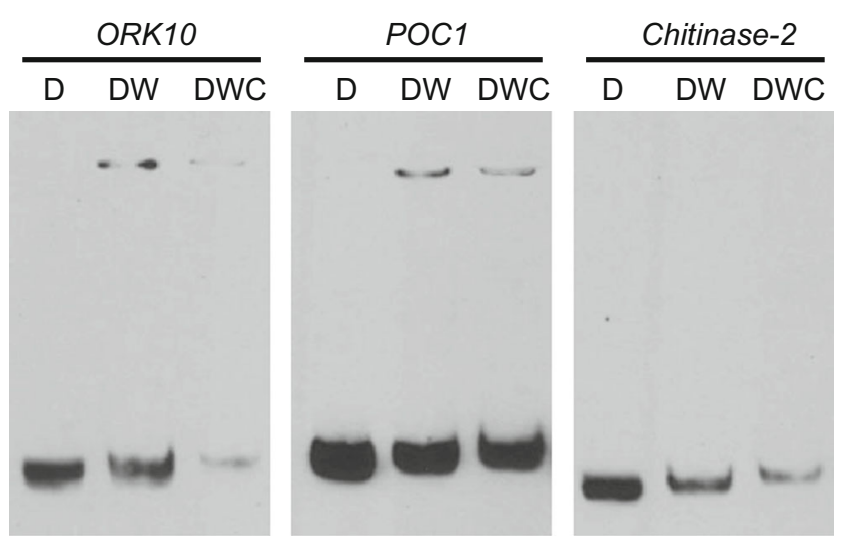

Fig. 4 Electrophoretic mobility shift assays. $D$ biotinylated DNA fragment, $W$ expressed TaWRKY53 protein, $C$ unlabeled competitor DNA

photosynthetic machinery from free-radical damage, or by quenching runaway ROS production during the hypersensitive response.

The Arabidopsis ortholog AtWRKY33 is phosphorylated by the stress-responsive mitogen-activated protein kinases
(MAPKs) MPK3 and MPK6 (Wan et al. 2004; Mao et al. 2011). Although orthologs of these MAPKs were not detected in our yeast two-hybrid assay, their inclusion in the working model (Fig. 5) of the WRKY53 transcriptional network is warranted; because of the evolutionary conservation of MAPK modules even between distantly related species (Asai et al. 2002; Hamel et al. 2006), WRKY53 remains a plausible target of such kinase signalling cascades in cereals, with its activity perhaps modulated by stress-responsive interactors such as the GST we identified.

We infer from our data that WRKY53 is able to transduce this wide range of stress-responsive signals to several downstream targets. Particularly prominent in our study is the involvement of the oxidative burst and genes forming part of the pathogen defence repertoire, including GST, ORK10 and POC1 (Fig. 4). ORK10/LRK10 is a receptor kinase important in the fungal resistance response of cereals (Feuillet et al. 1997; Cheng et al. 2002; Marcel et al. 2010), and

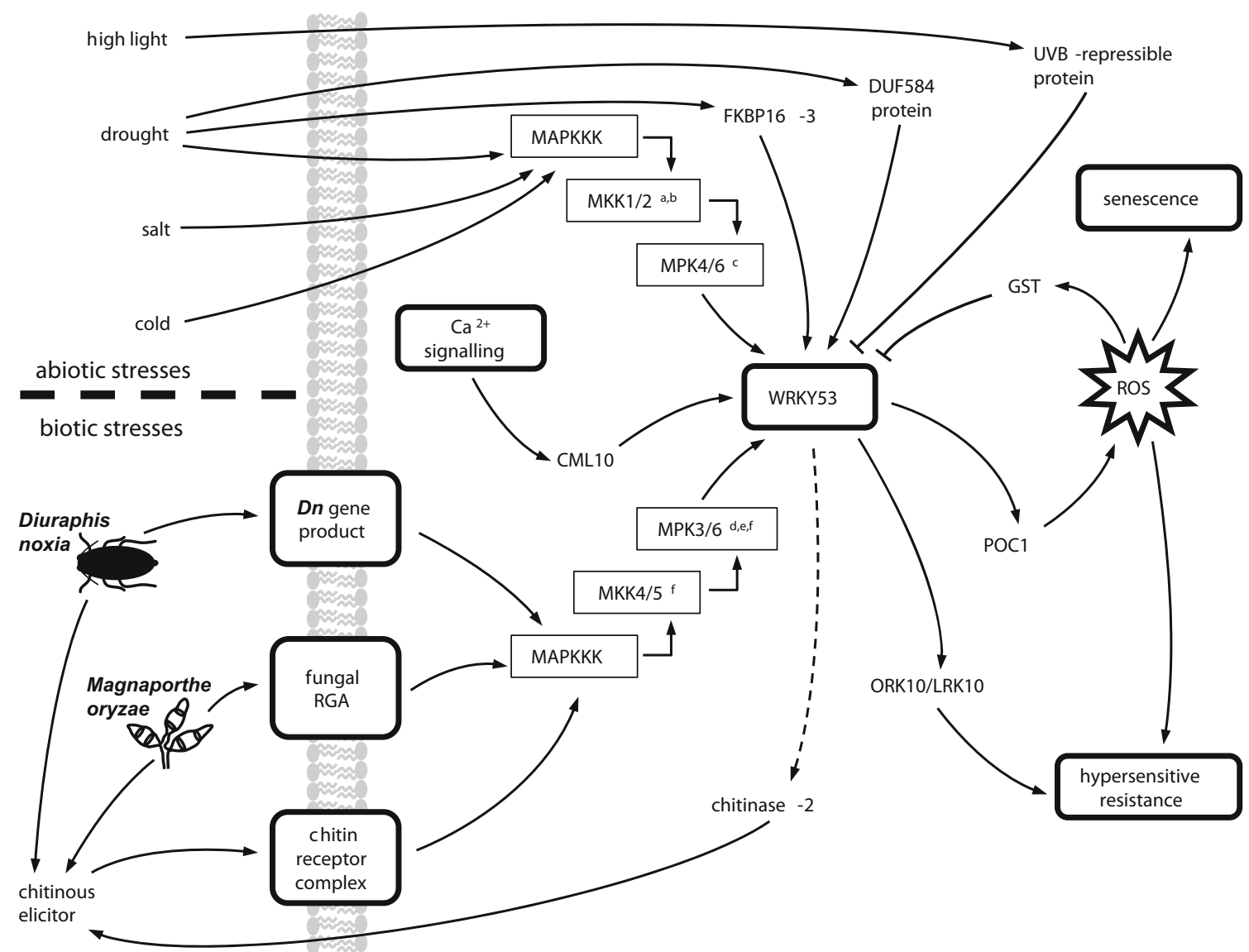

Fig. 5 A gene network for WRKY53 in cereals. The WRKY53 transcription factor is able to receive many types of stress inputs, from both abiotic and biotic pathways, and transduce those to an appropriate oxidative burst. Our study suggests that CML10, FKBP16-3, a DUF584 protein and a $U V B$-repressible protein are upstream regulatory components, whereas GST, ORK10/LRK10 and the peroxidase POC1 are downstream targets of WRKY53 regulation. ${ }^{\mathrm{a}}$ Ichimura et al. (1998), ${ }^{\mathrm{b}}$ Teige et al. (2004), ${ }^{\mathrm{c}}$ Qiu et al. (2008), ${ }^{\mathrm{d}} \mathrm{Wan}$ et al. (2004), ${ }^{\mathrm{e}} \mathrm{Mao}$ et al. (2011), ${ }^{\mathrm{f}}$ Asai et al. (2002) 
POC1 is an apoplastic peroxidase induced as part of the oxidative burst in response to aphids or pathogens (Young et al. 1995; Van der Westhuizen et al. 1998; Hilaire et al. 2001; Anguelova-Merhar et al. 2002). This is consistent with a regulatory role for $O S W R K Y 53$ during rice blast infection (Chujo et al. 2007) and TaWRKY53 during the resistance response to aphid attack (Van Eck et al. 2010) and implies that WRKY53 is a regulator of ROS release during the hypersensitive response (Fig. 5).

In summary, we have demonstrated that the gene structure and cis-acting regulatory elements of WRKY53 are highly conserved between wheat and rice, and report several novel genes that act as either upstream regulators affording WRKY53 responsiveness to various biotic and abiotic stress inputs, or downstream targets involved in oxidative responses to stress.

Acknowledgments We kindly thank Mariko Alexander for the assistance with the yeast-hybrid systems, Dr Marian Walhout (University of Massachusetts Medical School) for providing the yeast one-hybrid vectors and Dr Myron Bruce (USDA-ARS) for the helpful discussion. This study was supported by the US Department of Agriculture under the Cooperative Agreements USDA Contract No. 2009-34205-19960 and 2010-34205-21350 and Hatch Funds Project No. 644 to NL.

Open Access This article is distributed under the terms of the Creative Commons Attribution License which permits any use, distribution, and reproduction in any medium, provided the original author(s) and the source are credited.

\section{References}

Ahn JC, Kim D-W, You YN, Seok MS, Park JM, Hwang H, Kim B-G, Luan S, Park H-S, Cho HS (2010) Classification of rice (Oryza sativa L. japonica Nipponbare) immunophilins (FKBPs, CYPs) and expression patterns under water stress. BMC Plant Biol 10:253

Akimoto-Tomiyama C, Sakata K, Yazaki J, Nakamura K, Fujii F, Shimbo K, Yamamoto K, Sasaki T, Kishimoto N, Kikuchi S, Shibuya N, Minami E (2003) Rice gene expression in response to Nacetylchitooligosaccharide elicitor: comprehensive analysis by DNA microarray with randomly selected ESTs. Plant Mol Biol 52: 537-551

Anguelova-Merhar VS, Van der Westhuizen AJ, Pretorius ZA (2002) Intercellular chitinase and peroxidase activities associated with resistance conferred by gene $\operatorname{Lr} 35$ to leaf rust of wheat. J Plant Physiol 159:1259-1261

Asai T, Gena G, Plotnikova J, Willmann MR, Chiu WL, Gomez-Gomez L, Boller T, Ausubel FM, Sheen J (2002) MAP kinase signalling cascade in Arabidopsis innate immunity. Nature 415:977-983

Berman J, Eisenberg S, Tye B-K (1987) An agarose gel electrophoresis assay for the detection of DNA-binding activities in yeast cell extracts. Methods Enzymol 155:528-537

Botha A-M, Li Y, Lapitan NLV (2005) Cereal host interactions with Russian wheat aphid: a review. J Plant Interact 1:211-222

Botha A-M, Swanevelder ZH, Lapitan NLV (2010) Transcript profiling of wheat genes expressed during feeding by two different biotypes of Diuraphis noxia. Environ Entomol 39:1206-1231
Cheng DW, He S, Armstrong KC (2002) Modified expression of two receptor kinase genes in hexaploid oat (Avena sativa $\mathrm{L}$ ) on inoculation with crown rust. Physiol Mol Plant Pathol 61:281-288

Childs KL, Davidson RM, Buell CR (2011) Gene coexpression network analysis as a source of functional annotation for rice genes. PLoS One 6:e22196

Chittoor JM, Leach JE, White FF (1997) Differential induction of a peroxidase gene family during infection of rice by Xanthomonas oryzae pv oryzae. Mol Plant Microbe Interact 10:861-871

Christmann A, Moes D, Himmelbach A, Yang Y, Tang Y, Grill E (2006) Integration of abscisic acid signalling into plant responses. Plant Biol 8:314-325

Chujo T, Takai R, Akimoto-Tomiyama C, Ando S, Minami E, Nagamura Y, Kaku H, Shibuya N, Yasuda M, Nakashita H, Umemura K, Okada A, Okada K, Nojiri H, Yamane H (2007) Involvement of the elicitor-induced gene OsWRKY53 in the expression of defenserelated genes in rice. Biochim Biophys Acta 1769:497-505

Chujo T, Sugioka N, Masuda Y, Shibuya N, Takemura T, Okada K, Nojiri H, Yamane H (2009) Promoter analysis of the elicitor-induced WRKY gene OsWRKY53, which is involved in defense responses in rice. Biosci Biotechnol Biochem 73:1901-1904

Couldridge C, Newbury HJ, Ford-Lloyd B, Bale J, Pritchard J (2007) Exploring plant responses to aphid feeding using a full Arabidopsis microarray reveals a small number of genes with significantly altered expression. Bull Entomol Res 97:523-532

Cummins I, Dixon DP, Freitag-Pohl S, Skipsey M, Edwards R (2011) Multiple roles for plant glutathione transferases in xenobiotic detoxification. Drug Metab Rev 43:266-280

Deplancke B, Dupuy D, Vidal M, Walhout AJM (2004) A Gatewaycompatible yeast one-hybrid system. Genome Res 14:2093-2101

Dong N, Liu X, Lu Y, Du L, Xu H, Liu H, Xin Z, Zhang Z (2010) Overexpression of TaPIEP1, a pathogen-induced ERF gene of wheat, confers host-enhanced resistance to fungal pathogen Bipolaris sorokiniana. Funct Integr Gen 10:215-226

Drummond AJ, Ashton B, Buxton S, Cheung M, Cooper A, Duran C, Field M, Heled J, Kearse M, Markowitz S, Moir R, Stones-Havas S, Sturrock S, Thierer T, Wilson A (2011) Geneious 54 (http://www. geneious.com/) Biomatters Ltd, Auckland, New Zealand

Encinas-Villarejo S, Maldonado AM, Amil-Ruiz F, De los Santos B, Romero F, Pliego-Alfaro F, Muñoz-Blanco J, Caballero JL (2009) Evidence for a positive regulatory role of strawberry (Fragaria $\mathrm{x}$ ananassa) FaWRKY1 and Arabidopsis AtWRKY75 proteins in resistance. J Exp Bot 60:3043-3065

Eulgem T, Somssich IE (2007) Networks of WRKY transcription factors in defense signaling. Curr Opin Plant Biol 10:366-371

Feuillet C, Schachermayr G, Keller B (1997) Molecular cloning of a new receptor-like kinase gene encoded at the $\operatorname{Lr} 10$ disease resistance locus of wheat. Plant J 11:45-52

Fischer-Kilbienski I, Miao Y, Roitsch T, Zschiesche W, Humbeck K, Krupinska K (2010) Nuclear targeted AtS40 modulates senescence associated gene expression in Arabidopsis thaliana during natural development and in darkness. Plant Mol Biol 73:379-390

Galon Y, Nave R, Boyce JM, Nachmias D, Knight MR, Fromm H (2008) Calmodulin-binding transcription activator (CAMTA) 3 mediates biotic defense responses in Arabidopsis. FEBS Lett 582:943-948

Gill SS, Tuteja N (2010) Reactive oxygen species and antioxidant machinery in abiotic stress tolerance in crop plants. Plant Physiol Biochem 48:909-930

Gollan PJ, Bhave M (2010) Genome-wide analysis of genes encoding FK506-binding proteins in rice. Plant Mol Biol 72:1-16

Hahn K, Strittmatter G (1994) Pathogen-defence gene prp 1-1 from potato encodes an auxin-responsive glutathione $S$-transferase. Eur J Biochem 226:619-626

Hamel L-P, Nicole M-C, Sritubtimb S, Morency M-J, Ellis M, Ehlting J, Beaudoin N, Barbazuk B, Klessig D, Lee J, Martin G, Mundy J, 
Ohashi Y, Scheel D, Sheen J, Xing T, Zhang S, Seguin A, Ellis BE (2006) Ancient signals: comparative genomics of plant MAPK and MAPKK gene families. Trends Plant Sci 11:192-198

Hellman LM, Fried MG (2007) Electrophoretic mobility shift assay (EMSA) for detecting protein-nucleic acid interactions. Nat Protoc 2:1849-1861

Higo K, Ugawa Y, Iwamoto M, Korenaga T (1999) Plant cis-acting regulatory DNA elements (PLACE) database: 1999. Nucleic Acids Res 27:297-300

Hilaire E, Young SA, Willard LH, McGee JD, Sweat T, Chittoor JM, Guikema JA, Leach JE (2001) Vascular defense responses in rice: peroxidase accumulation in xylem parenchyma cells and xylem wall thickening. Mol Plant Microbe Interact 14:1411-1419

Hosack DA, Dennis G, Sherman BT, Lane HC, Lempicki RA (2003) Identifying biological themes within lists of genes with EASE. Genome Biol 4:R70

Huang DW, Sherman BT, Lempicki RA (2008) Systematic and integrative analysis of large gene lists using DAVID bioinformatics resources. Nat Protoc 4:44-57

Ichimura K, Mizoguchi T, Irie K, Morris P, Giraudat J, Matsumoto K, Shinozaki K (1998) Isolation of ATMEKK1 (a MAP kinase kinase kinase)-interacting proteins and analysis of a MAP kinase cascade in Arabidopsis. Biochem Biophys Res Commun 253:532-543

Izaguirre MM, Scopel AL, Baldwin IT, Ballare CL (2003) Convergent responses to stress solar ultraviolet-B radiation and Manduca sexta herbivory elicit overlapping transcriptional responses in field-grown plants of Nicotiana longiflora. Plant Physiol 132:1755-1767

Jakobsson P-J, Morgenstern R, Mancini J, Ford-Hutchinson A, Persson B (1999) Common structural features of $M A P E G$ - a widespread superfamily of membrane associated proteins with highly divergent functions in eicosanoid and glutathione metabolism. Protein Sci 8: 689-692

Kaloshian I, Walling LL (2005) Hemipterans as plant pathogens. Annu Rev Phytopathol 43:491-521

Krupinska K, Haussühl K, Schäfer A, Van der Kooij TAW, Leckband G, Lörz H, Falk J (2002) A novel nucleus-targeted protein is expressed in barley leaves during senescence and pathogen infection. Plant Physiol 130:1172-1180

Lai Z, Wang F, Zheng Z, Fan B, Chen Z (2011) A critical role of autophagy in plant resistance to necrotrophic fungal pathogens. Plant J 66:953-968

Lee I, Seo Y, Coltrane D, Hwang S, Oh T, Marcotte EM, Ronald PC (2011) Genetic dissection of the biotic stress response using a genome-scale gene network for rice. Proc Natl Acad Sci U S A 108:18548-18553

Lescot M, Déhais P, Thijs G, Marchal K, Moreau Y, Van de Peer Y, Rouzé P, Rombauts S (2002) PlantCARE, a database of plant cis-acting regulatory elements and a portal to tools for in silico analysis of promoter sequences. Nucleic Acids Res 30:325-327

Lieberherr D, Wagner U, Dubuis P-H, Métraux J-P, Mauch F (2003) The rapid induction of glutathione $S$-transferases AtGSTF2 and AtGSTF6 by avirulent Pseudomonas syringae is the result of combined salicylic acid and ethylene signaling. Plant Cell Physiol 44: $750-757$

Lippok B, Birkenbihl RP, Rivory G, Brümmer J, Schmelzer E, Logemann E, Somssich IE (2007) Expression of AtWRKY33 encoding a pathogen- or PAMP-responsive WRKY transcription factor is regulated by a composite DNA motif containing W box elements. Mol Plant Microbe Interact 20:420-429

Mao G, Meng X, Liu Y, Zheng Z, Chen Z, Zhang S (2011) Phosphorylation of a WRKY transcription factor by two pathogenresponsive MAPKs drives phytoalexin biosynthesis in Arabidopsis. Plant Cell 23:1639-1653

Marcel S, Sawers R, Oakeley E, Angliker H, Paszkowski U (2010) Tissue-adapted invasion strategies of the rice blast fungus Magnaporthe oryzae. Plant Cell 22:3177-3187
Moloi MJ, Van der Westhuizen AJ (2008) Antioxidative enzymes and the Russian wheat aphid (Diuraphis noxia) resistance response in wheat (Triticum aestivum). Plant Biol 10:403-407

Moran PJ, Thompson GA (2001) Molecular responses to aphid feeding in Arabidopsis in relation to plant defense pathways. Plant Physiol 125:1074-1085

Nakayama M, Kikuno R, Ohara O (2002) Protein-protein interactions between large proteins: two-hybrid screening using a functionally classified library composed of long cDNAs. Genome Res 12:1773-1784

Ni X, Quisenberry SS (2003) Possible roles of esterase, glutathione $S$ transferase, and superoxide dismutase activities in understanding aphid-cereal interactions. Entomol Exp Appl 108:187-195

Ni X, Quisenberry SS, Markwell J, Heng-Moss T, Higley L, Baxendale F, Sarath G, Klucas R (2001) In vitro enzymatic chlorophyll catabolism in wheat elicited by cereal aphid feeding. Entomol Exp Appl 101:159-166

Niño-Liu DO, Darnielle L, Bogdanove AJ (2005) A simple method of mass inoculation of rice effective for both pathovars of Xanthomonas oryzae, and the construction of comparable sets of host cDNA libraries spanning early stages of bacterial leaf blight and bacterial leaf streak. J Phytopathol 153:500-504

Olbrich M, Betz G, Gerstner E, Langebartels C, Sandermann H, Ernst D (2005) Transcriptome analysis of ozone-responsive genes in leaves of European beech (Fagus sylvatica L). Plant Biol 7:670-676

Panavas T, Sanders C, Butt TR (2009) SUMO fusion technology for enhanced protein production in prokaryotic and eukaryotic epxression systems. In: Ulrich HD (ed) SUMO protocols, vol 497. Springer Science, New York, pp 303-317

Park S-J, Huang Y, Ayoubi P (2006) Identification of expression profiles of sorghum genes in response to greenbug phloemfeeding using cDNA subtraction and microarray analysis. Planta 223:932-947

Popescu SC, Popescu GV, Bachan S, Zhang Z, Seay M, Gerstein M, Snyder M, Dinesh-Kumar SP (2007) Differential binding of calmodulin-related proteins to their targets revealed through high-density Arabidopsis protein microarrays. Proc Natl Acad Sci U S A 104:4730-4735

Qiu J-L, Fiil BK, Petersen K et al (2008) Arabidopsis MAP kinase 4 regulates gene expression through transcription factor release in the nucleus. EMBO J 27:2214-2221

Ramamoorthy R, Jiang S-Y, Kumar N, Venkatesh PN, Ramachandran S (2008) A comprehensive transcriptional profiling of the WRKY gene family in rice under various abiotic and phytohormone treatments. Plant Cell Physiol 49:865-879

Ramonell K, Berrocal-Lobo M, Koh S, Wan J, Edwards H, Stacey G, Somerville S (2005) Loss-of-function mutations in chitin responsive genes show increased susceptibility to the powdery mildew pathogen Erysiphe cichoracearum. Plant Physiol 138:1027-1036

Reddy VS, Reddy ASN (2004) Proteomics of calcium-signaling components in plants. Phytochemistry 65:1745-1776

Rodriguez PA, Bos JIB (2013) Toward understanding the role of aphid effectors in plant infestation. Mol Plant Microbe Interact 26:25-30

Rushton PJ, Somssich IE (1998) Transcriptional control of plant genes responsive to pathogens. Curr Opin Plant Biol 1:311-315

Rushton PJ, Somssich IE, Ringler P, Shen QJ (2010) WRKY transcription factors. Trends Plant Sci 15:247-258

Shimono M, Sugano S, Nakayama A, Jiang C-J, Ono K, Toki S, Takatsuji H (2007) Rice WRKY45 plays a crucial role in benzothiadiazoleinducible blast resistance. Plant Cell 19:2064-2076

Smith CM, Boyko EV (2007) The molecular bases of plant resistance and defense responses to aphid feeding: current status. Entomol Exp Appl 122:1-16

Smith CM, Liu X, Wang LJ, Liu X, Chen M-S, Starkey S, Bai J (2010) Aphid feeding activates expression of a transcriptome of oxylipinbased defense signals in wheat involved in resistance to herbivory. J Chem Ecol 36:260-276 
Teige M, Scheikl E, Eulgem T, Doczi R, Ichimura K, Shinozaki K, Dangl JL, Hirt H (2004) The MKK2 pathway mediates cold and salt stress in Arabidopsis. Mol Cell 15:141-152

Turck F, Zhou A, Somssich IE (2004) Stimulus-dependent, promoterspecific binding of transcription factor WRKY1 to its native promoter and the defense-related gene PcPR1-1 in parsley. Plant Cell $16: 2573-2585$

Van der Westhuizen A, Qian X-M, Botha A-M (1998) Differential induction of apoplastic peroxidase and chitinase activities in susceptible and resistant wheat cultivars by Russian wheat aphid infestation. Plant Cell Rep 18:132-137

Van Eck L, Schultz T, Leach JE, Scofield SR, Peairs FB, Botha A-M, Lapitan NLV (2010) Virus-induced gene silencing of WRKY53 and an inducible phenylalanine ammonia-lyase in wheat reduces aphid resistance. Plant Biotechnol J 8: $1023-1032$
Wan J, Zhang S, Stacey G (2004) Activation of a mitogen-activated protein kinase pathway in Arabidopsis by chitin. Mol Plant Pathol 5:125-135

Wang H, Hao J, Chen X, Hao Z, Wang X, Lou Y, Peng Y, Guo Z (2007) Overexpression of rice WRKY89 enhances ultraviolet B tolerance and disease resistance in rice plants. Plant Mol Biol 65:799-815

Wu H, Ni Z, Yao Y, Guo G, Sun Q (2008) Cloning and expression profiles of 15 genes encoding WRKY transcription factor in wheat (Triticum aestivum L). Prog Nat Sci 18:697-705

Young SA, Guo AA, Guikema JA, White FF, Leach JE (1995) Rice cationic peroxidase accumulates in xylem vessels during incompatible interactions with Xanthomonas oryzae pv oryzae. Plant Physiol 107:1333-1341

Zhu X, Liu S, Meng C, Qin L, Kong L, Xia G (2013) WRKY transcription factors in wheat and their induction by biotic and abiotic stress. Plant Mol Biol Report 31:1053-1067 